\title{
Uniformity of Metabolic Enzymes Within Individual Motor Units
}

\author{
Patti M. Nemeth, Lata Solanki, Debra A. Gordon, ${ }^{*}$ Thomas M. Hamm, ${ }^{*}$ Robert M. Reinking, ${ }^{*}$ and \\ Douglas G. Stuart ${ }^{*}$ \\ Departments of Neurology and of Anatomy and Neurobiology, Washington University School of Medicine, St. Louis, \\ Missouri 63110, and the *Department of Physiology, University of Arizona Center for Health Sciences, Tucson, \\ Arizona 85724
}

Individual muscle fibers of $\mathbf{1 0}$ motor units from the tibialis posterior muscle of cat were identified by glycogen depletion techniques, characterized for histochemical type, diameter, and intramuscular locations, and analyzed by quantitative biochemical methods. Four enzymes, representing different energy-yielding pathways, were quantitatively assayed in muscle fibers belonging to motor units selected from each of the three major physiological types. All four enzymes demonstrated identical activities among fibers within a motor unit, while showing up to 11-fold differences among fibers belonging to different motor units. Moreover, fibers within a single motor unit, but of substantially different diameters, were nevertheless homogeneous in specific enzyme activities.

The force-generating capability of muscle depends upon the enzymatic protcins that provide encrgy. These and other biochemical characteristics are known to be regulated by the functional demands imposed on the muscle by means of its innervation (e.g., Buller et al., 1960; Vrbová et al., 1978). Implicit in the idea originally formulated by Sherrington (1925), that the motor unit forms the smallest functional component of skeletal muscle controlled by the nervous system, is the concept of biochemical uniformity among the unit's muscle fibers. The test of this concept was made possible by the glycogen depletion technique, proposed by Krnjevic and Miledi (1958) and first used by Edström and Kugelberg (1968), which involves electrical activation of single motor units until their constituent muscle fibers are depleted of glycogen, thereby permitting histochemical identification and characterization of the fibers of a single motor unit. Analysis of motor units identified by glycogen depletion has revealed that both the staining intensities for various enzymes (Burke et al., 1971; Edström and Kugelberg, 1968) and the quantitative levels of malate dehydrogenase activities (Nemeth et al., 1981) were virtually identical within fibers of individual motor units.

The generalization of the concept that a given motor unit contains biochemically identical fibers has recently been questioned on the basis of results on enzymes measured by quantitative histochemistry (Roy et al., 1984). A wide range of values was reported among motor unit fibers for succinate dehydrogenase and $\alpha$-glycerophosphate dehydrogenase. If, indeed, motor units are composed of metabolically dissimilar fibers, it would

Received May 20, 1985; revised Aug. 23, 1985; accepted Aug. 25, 1985.

We are grateful to J. Park, L. L. Rankin, S. Vanden-Noven, K. Volz, and P. Pierce for technical assistance and to Drs. O. H. Lowry and R. Wilkinson for reading the manuscript. The work was supported by U.S.P.H.S. Grants HL 0724, NS 18387 , NS 20544, and NS/AM 17887 and by the Muscular Dystrophy Association.

Correspondence should be addressed to Dr. Patti M. Nemeth, Department of Neurology, Washington University School of Medicine, 660 South Euclid Avenue, Box 8111 , St. Louis, MO 63110.

Copyright (C) 1986 Society for Neuroscience 0270-6474/86/030892-07\$02.00/0 have far-reaching implications with respect to neural control and the functional unity assumed for the motor unit (for review, see Burke, 1981; Stuart et al., 1984). The determination of this important issue requires the high resolution afforded by microanalytical techniques (Lowry and Passonneau, 1972). However, until now the isolation of motor unit fibers for this type of analysis was so laborious as to be incapable of providing sufficient material for unambiguous results at high levels of confidence.

Therefore, a technique was developed to facilitate the microanalytic approach on large samples of motor units. It is an in situ histochemical identification of glycogen-depleted fibers that allows their subsequent dissection for microchemical analysis from unstained serial transverse sections. The increased ease and speed provided by the technique permits the rigorous asscssment of large numbers of motor units and, in addition, provides information about the individual fiber size, histochemical characteristics, and intramuscular location. Our results give further evidence for general metabolic homogeneity of muscle fibers within a single motor unit, even with extreme interfiber size differences. Brief accounts of some of these data have bcen published (Nemeth et al., 1984; and in press).

\section{Materials and Methods}

Mixed-breed adult male cats $(2-3 \mathrm{~kg})$ were confined to a standard-size cage for $21 \mathrm{~d}$ prior to experimentation to ensure some similarity of exercise exposure. For the last $3 \mathrm{~d}$ of confinement, $2.2 \%$ D-glucose was added to their drinking water in order to glycogen-load the muscle.

Under gaseous anesthesia (halothane-nitrous oxide-oxygen), one hindlimb was completely denervated except for the tibialis posterior muscle. A strain ring was attached to the severed distal tendon. The vertebral column was stabilized in a Göteborg-type spinal frame, and a laminectomy was performed to expose the appropriate ventral root (L7). The surgical and electrophysiological procedures have been previously described in detail (McDonagh et al., 1980).

A single motor unit was activated by square wave electrical pulses (0.1 msec duration) applied to a small group of motor axons split from the 17 ventral root. A neurogram was obtained from a recording electrode positioned on the nerve to the tibialis posterior muscle. It provided the input to a signal averager triggered by the ventral root stimulus train (at $10 \times$ threshold). The average action potential extracted from the neurogram served as proof of single motor unit isolation, this being a much more reliable procedure than observance of the simultaneously measured electromyogram and/or twitch force.

On the basis of twitch contraction time, a profile of unfused tetanus ("sag" test), and a 2 min fatigue test (for a review, see Burke, 1981; McDonagh et al., 1980), motor units were classified as fast-contracting and fatigable (FF), fast-contracting and fatigue-resistant (FR), and slowcontracting fatigue-resistant (S). Motor units were selected to cover the wide range of functional types previously identified in the cat tibialis posterior muscle (McDonagh et al., 1980).

The motor unit was depleted of glycogen by electrical activation of its motor axon with $1 / \mathrm{sec} 40 \mathrm{~Hz}$ stimulus trains of $330 \mathrm{msec}$ duration. The FF units, which lost appreciable tension within a few minutes, were 

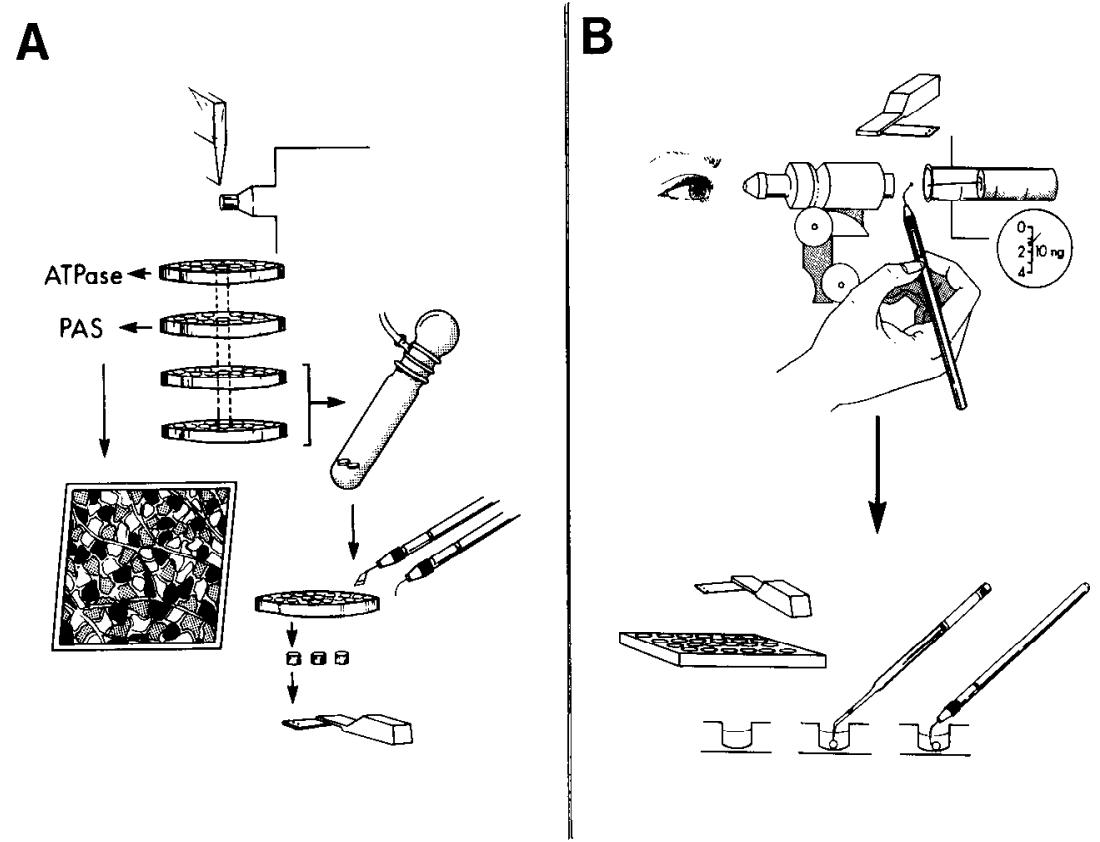

Figure 1. In situ histochemical identification of fibers for microanalytical biochemistry. $A$, Muscles are mounted in embedding medium on blocks with fibers oriented longitudinally, then quickly frozen in liquid nitrogen near its freezing point. Serial transverse sections are cut with a cryostat microtome. Alternate sections are either stained for histochemistry or prepared for biochemistry. The latter are lyophilized under vacuum. Photomicrographs of stained sections are used as maps to identify fibers in the adjacent, unstaincd lyophilized sections. Individual fiber segments are isolated by microdissection techniques. $B$, Fiber segments are weighed on a quartz fiber torsion balance and subjected to assays in microliter volumes pipetted under oil to avoid evaporation.

stimulated for $15 \mathrm{~min}$; the FR and $\mathrm{S}$ units were stimulated for a standard $1 \mathrm{hr}$, irrespective of the extent to which their force output declined. Immediately after the glycogen depletion procedure, the muscle was removed, quickly frozen (within $60 \mathrm{sec}$ ) in liquid nitrogen that had been cooled almost to its freezing point $\left(-210^{\circ} \mathrm{C}\right)$, and stored at $-70^{\circ} \mathrm{C}$. The frozen muscle was divided into blocks (of approximatcly $5 \times 5 \times 12$ $\mathrm{mm}$ ) and mounted in embedding medium, with the fibers oriented longitudinally.

Schematic illustrations show the methods of fiber procurement (Fig. $1 A$ ) and of microanalysis (Fig. $1 B$ ). Serial transverse sections of 16-18 $\mu \mathrm{m}$ were cut on a microtome in a cryostat $\left(-20^{\circ} \mathrm{C}\right)$. Alternate sections were processed for myosin ATPase stain after preincubation at $\mathrm{pH} 4.5$ to identify myosin fiber types (Brooke and Kaiser, 1970), for periodic acid-Schiff reaction to identify glycogen-depleted fibers (Nemeth et al., 1981), and for microanalytical biochemistry. These last sections were lyophilized at $-38^{\circ} \mathrm{C}$ at a pressure of less than $10^{-2}$ Torr for $24 \mathrm{hr}$. Photographs of the stained sections were used as maps to identify the motor unit fibers in the adjacent unstained lyophilized sections. The fibers in the latter sections were teased out under a stereomicroscope with finc handmade instruments. Multiple samples of the same cell were obtained from sequential unstained sections. The fiber samples, 30-40 $\mu \mathrm{m}$ in diameter and $16-18 \mu \mathrm{m}$ thick, were weighed precisely on a quartz fiber torsion balance. Samples were added to microliter volumes (5-10 $\mu$ l) of assay reagent that had been pipetted under purified mineral oil to avoid evaporation. All assays ultimately yielded a fluorescent pyridine nucleotide product, which was measured with a filter fluorometer. Further details of the analytic methods are provided by Lowry and Passonneau (1972). Photomicrographs of tissue samples before and after dissection are shown in Figure 2.

Four enzymes, chosen to represent major energy-yielding pathways, were assayed according to previously described methods: adenylokinase for high-energy phosphate metabolism, lactate dehydrogenase for anaerobic glycolysis, $\beta$-hydroxylacyl-CoA dehydrogenase for fatty acid oxidation (Lowry et al., 1978), and malate dehydrogenase for the citric acid cycle (Hintz et al., 1980). Enzyme activities are expressed in mol/ $\mathrm{kg}$ dry weight $/ \mathrm{hr} \pm \mathrm{SD}$ of duplicate or triplicate samples.

To determine whether the interfiber variability of enzyme activity was greater than that attributable to intrafiber variability, a one-way analysis of variance was performed for each motor unit. The analysis provided an $F$ ratio, the ratio of the mean square error between fibers to that within fibers. The $F$ ratio has been used in our study as an index of interfiber enzyme variability (see Table 1); a value significantly greater than 1.0 would indicate that fibers constituting the motor unit differ in their mean activity for the particular enzyme.

\section{Results}

\section{Histochemistry}

Mosaic patterns of fibers depleted of glycogen were found in all 10 motor units studied, including type $S$ units exhibiting no reduction of force in the $1 \mathrm{hr}$ fatigue test. In addition, a correspondence was noted between the histochemistry of the unit fibers and the physiologically determined motor unit type in each case: The $S$ unit fibers stained dark, the FR fibers stained light, and the FF fibers stained intermediately with myosin ATPase stain, as has been reported by other laboratories (Burke, 1981; Kugelberg, 1973).

The periodic acid-Schiff stain showed some peculiarities, in that not all experiments resulted in depletion of a single fiber type. There were muscles with areas of depleted fibers of all types. There were also cases in which many $\mathbf{S}$ units were so low in glycogen staining, despite glycogen loading, that they could not be distinguished from depleted FF units; this problem was usually corrected by glycogen enhancement with a uridine diphosphoglucose treatment, as previously described (Nemeth et al., 1981). In addition, on general inspection there were occasional depleted fibers of types other than the predominant type of motor unit, as well as random depletions in contralateral control muscles. Although aberrant fiber depletions may represent an inherent limitation to the glycogen depletion technique, they are, fortunately, easily recognized in serial transverse sections and, thus, avoidable. In this study, muscle fibers were examined in serial sections to ensure that depleted fibers were of the same myosin ATPase type, and thereby to eliminate the possibility of false-staining fibers with periodic acid-Schiff. In some experiments, examination revealed that several fibers of more than one histochemical type were depleted, and these muscles were eliminated from the study. In the subsequent selection of fibers for each of the remaining 10 motor units, there were no cases in which individual fibers had to be eliminated, which indicated that random aberrant depletions are relatively rare.

It was not possible to evaluate an entire fiber population of tibialis posterior muscle for the complete motor unit owing to 


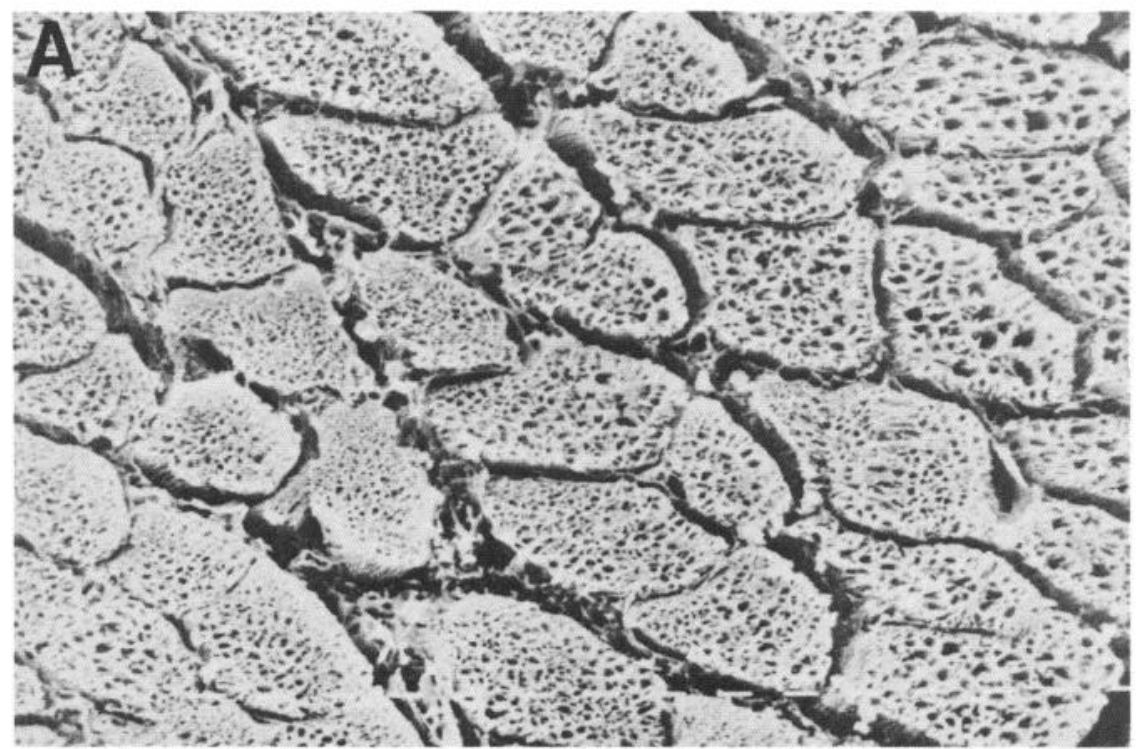

Figure 2. Scanning electron micrographs of transverse section of cat tibialis posterior muscle. Micrographs were made with a Philips 501 scanning electron microscope at a $30^{\circ}$ tilt (with resulting foreshortening of the $y$ axis), 15 $\mathrm{kV}, 200 \AA$ scanning spot size, following ion etching of freeze-dried samples with pure gold (Plasma Coater, Denton Vacuum). $A$, An intact transverse section is shown at $\times 320$; calibration bar $=10$ $\mu \mathrm{m} . B$, A single fiber dissected from the above transverse section, illustrating the lack of extracellular material that might otherwise affect the precision of quantitative analysis. Fenestrations produced in the freezing process make enzymes throughout the sample accessible to the assay reagents. Magnification, $\times 960$; calibration bar, $6.7 \mu \mathrm{m}$.

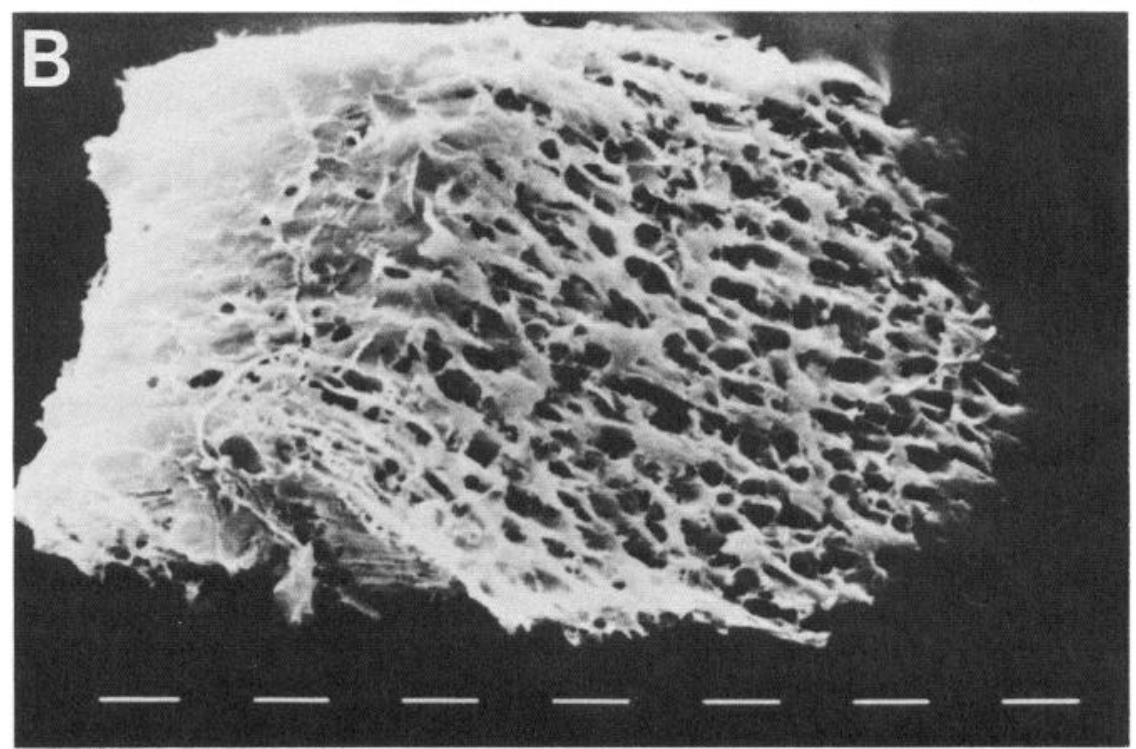

its bipennate structure; however, the structure provided other advantages. Motor unit fibers may be widely distributed over the muscle, and therefore were selected, in some cases, from spatially distant extremes of the muscle. It was also recognized that differences occur in fiber diameters, apparently in relation to the proximity of the fibers to the central tendon. In one case, extreme differences in the sizes of depleted fibers of the same myosin ATPase type occurred in the same block (Fig. 3), thus providing an opportunity to test for biochemical uniformity in morphologically different fibers of the same unit (see Results).

\section{Quantitative enzyme activities}

The tibialis posterior muscle was examined for enzyme continuity along the length of individual fibers (intrafiber variability) in order to validate the use of duplicate determinations for specific cellular enzyme activity. Sixteen randomly selected fibers were dissected from longitudinal fiber bundles, using the techniques of Essén et al. (1975). Figure 4 shows activities of the enzymes under study from segments cut consecutively from individual long fibers. The results indicate that fibers rarely vary by more than $10 \%$ (coefficient of variation) along approximately
$450 \mu \mathrm{m}$ fiber lengths. Interestingly, the variations differ for different enzymes and, in general, vary less in cytosolic enzymes (lactate and malate dehydrogenase and adenylokinase) than in the membrane-bound enzyme ( $\beta$-hydroxyacyl-CoA dehydrogenase), a finding that supports the earlier conclusion of Hintz et al. (1984). Duplicate determinations in the motor unit study were, therefore, considered representative for a particular fiber if the difference between the two measurements was equal to or less than the value obtained for intrafiber variation of the enzyme.

Table 1 gives the mean activity, SD, and $F$ ratio for each of the four enzymes measured on the fibers of each motor unit. Coefficients of variation among fibers were less than $10 \%$ in all motor units and for each enzyme except $\beta$-hydroxyacyl-CoA dehydrogenase, which exhibits, on the average, greater variation both within (Fig. 4) and among (Table 1) the individual fibers. This level of variability between fibers of individual motor units was no greater than that attributable to intrafiber variability, as shown by an analysis of variance. The $F$ ratios are all near 1.0, which clearly indicates uniformity in all of the motor units for all enzymes (see Discussion). 


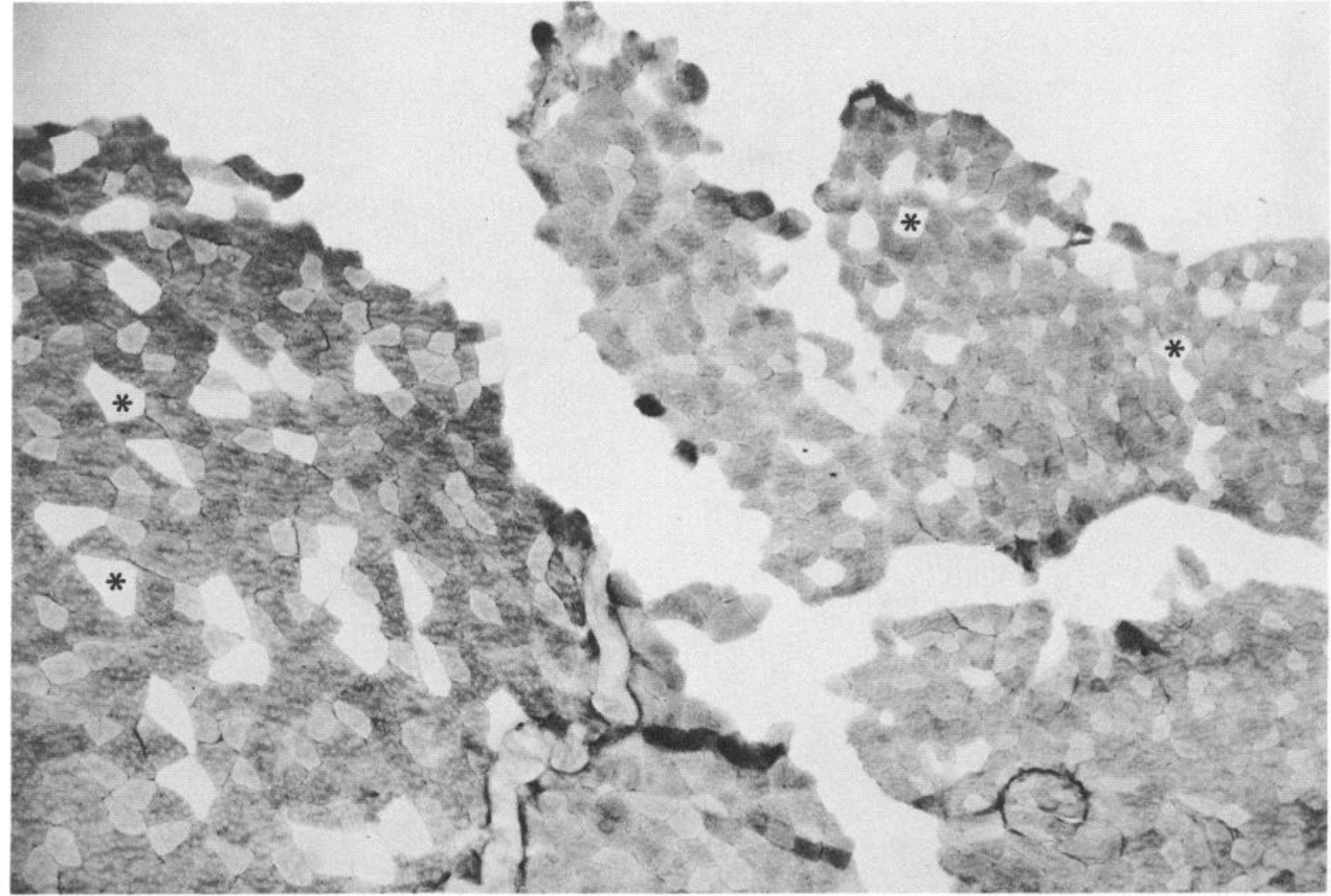

Figure 3. Transverse section of cat tibialis posterior muscle stained for periodic acid-Schiff after electrical activation of a single motor unit. Depleted muscle fibers of extemely different sizes (four marked with asterisks) stain the same for myosin ATPase in serial transverse sections and presumably belong to the same motor unit.

In one motor unit, it was possible to test biochemical properties of constituent fibers of extremely different sizes. Four fibers were selected from the motor unit shown in Figure 3. The individual fiber data are given in Table 2. The $F$ ratios indicate significant interfiber enzymatic uniformity, which is independent of fiber size. Thus they provide the clearest evidence for functional unity throughout the motor unit. Different fiber diameters might reflect tapering of these fibers near the tendon.

Table 1. Comparison of enzyme activities ( $\mathrm{mol} / \mathrm{kg} \mathrm{dry} \mathrm{weight/hr)} \mathrm{within} \mathrm{and} \mathrm{among} \mathrm{single} \mathrm{fibers} \mathrm{of} \mathrm{individual} \mathrm{motor} \mathrm{units} \mathrm{of} \mathrm{physiologically}$ determined types

\begin{tabular}{|c|c|c|c|c|c|c|c|c|}
\hline $\begin{array}{l}\text { Motor } \\
\text { unit } \\
\text { type }\end{array}$ & \multicolumn{2}{|c|}{ Adenylokinase } & \multicolumn{2}{|c|}{ Lactate dehydrogenase } & \multicolumn{2}{|c|}{ Malate dehydrogenase } & \multicolumn{2}{|c|}{$\begin{array}{l}\beta \text {-Hydroxyacyl-CoA } \\
\text { dehydrogenase }\end{array}$} \\
\hline FF & $228 \pm 17$ & $(0.71 / 3)$ & $234 \pm 6$ & $(0.89 / 8)$ & $3.3 \pm 0.2$ & $(0.81 / 8)$ & $1.4 \pm 0.1$ & $(1.69 / 8)$ \\
\hline $\mathrm{FF}$ & $211 \pm 9$ & $(1.00 / 3)$ & $251 \pm 10$ & $(2.55 / 7)$ & $3.1 \pm 0.2$ & $(2.33 / 7)$ & $1.4 \pm 0.1$ & $(0.53 / 8)$ \\
\hline $\mathrm{FF}$ & $246 \pm 6$ & $(0.72 / 3)$ & $294 \pm 6$ & $(0.29 / 8)$ & $4.0 \pm 0.2$ & $(1.98 / 8)$ & $1.3 \pm 0.1$ & $(0.39 / 8)$ \\
\hline FF & $238 \pm 11$ & $(1.19 / 7)$ & $160 \pm 7$ & $(0.98 / 8)$ & $4.6 \pm 0.2$ & $(3.31 / 6)$ & $1.4 \pm 0.1$ & $(0.18 / 6)$ \\
\hline FR & $187 \pm 15$ & $(0.79 / 4)$ & $251 \pm 7$ & $(0.28 / 3)$ & $14.1 \pm 0.4$ & $(0.86 / 3)$ & $9.8 \pm 0.6$ & $(1.48 / 3)$ \\
\hline FR & $176 \pm 3$ & $(0.20 / 3)$ & $319 \pm 13$ & $(1.93 / 4)$ & $13.2 \pm 0.8$ & $(1.11 / 6)$ & $7.4 \pm 0.3$ & $(1.04 / 2)$ \\
\hline s & $33 \pm 4$ & $(3.35 / 5)$ & $41.9 \pm 2.6$ & $(1.71 / 7)$ & $17.3 \pm 0.2$ & $(0.17 / 5)$ & $9.6 \pm 0.7$ & $(0.71 / 7)$ \\
\hline S & $33 \pm 2$ & $(0.39 / 7)$ & $30.7 \pm 2.6$ & $(1.20 / 7)$ & $15.4 \pm 0.8$ & $(1.33 / 4)$ & $10.0 \pm 0.4$ & $(0.51 / 4)$ \\
\hline
\end{tabular}

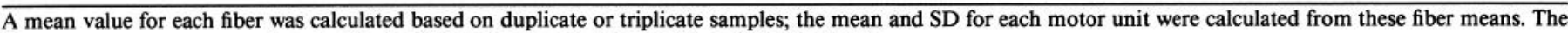
four enzymes were measured on two to eight fibers from each motor unit, as indicated by $N$.

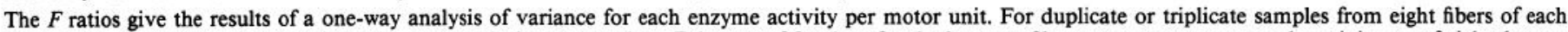

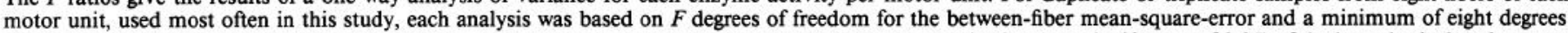

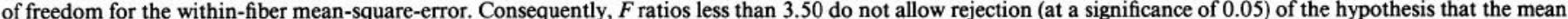

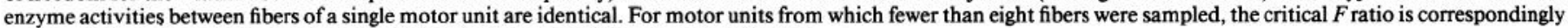
greater. In the values given above, none of the $F$ ratios are significantly greater than 1 .

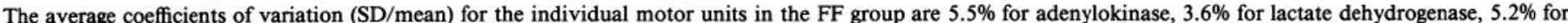

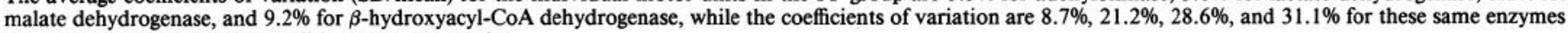
when computed between the different FF motor units. 

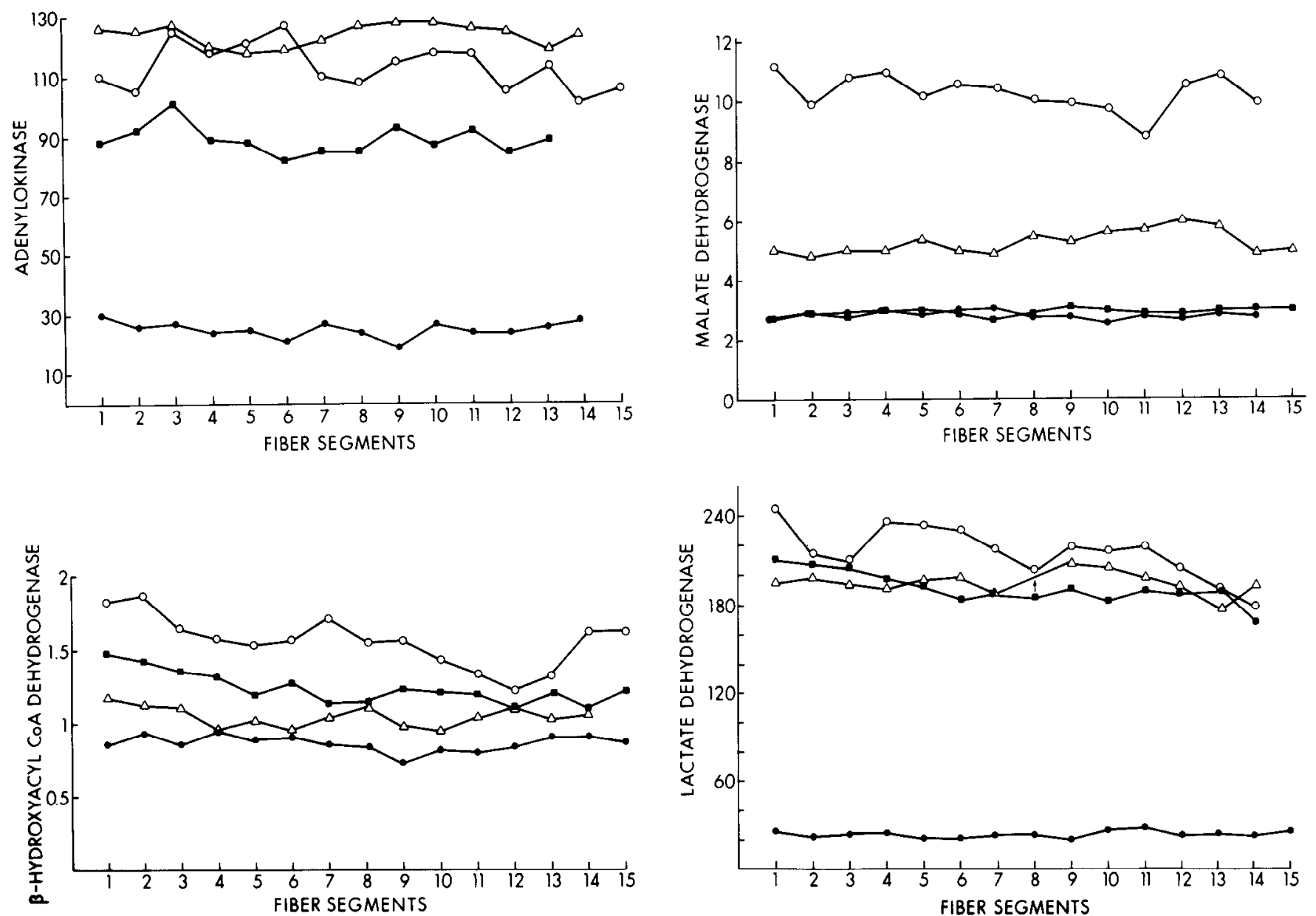

Figure 4. Enzyme activities in sequential segments of fibers dissected from bundles of cat tibialis posterior muscle. Different symbols identify four individual fibers. [For adenylokinase: $\Delta=124 \pm 3.5(2.9 \%) ; O=113 \pm 7.5(6.6 \%) ; \mathbf{v}=89 \pm 4.7(5.2 \%) ; \bullet=25 \pm 2.8(11.1 \%)$. For malate dehydrogenase: $O=10.2 \pm 0.8(7.4 \%) ; \boldsymbol{\square}=2.9 \pm 0.1(4.1 \%) ; \Delta=5.3 \pm 0.4(6.7 \%) ; \bullet=2.8 \pm 0.1(4.9 \%)$. For $\beta$-hydroxyacyl-CoA dehydrogenase: $O=1.57 \pm 0.17(11.0 \%) ; \square=1.24 \pm 0.11(8.67 \%) ; \Delta=1.05 \pm 0.07(6.6 \%) ;=0.87 \pm 0.05(6.2 \%)$. For lactate dehydrogenase: $O=217 \pm 17$ $(7.8 \%) ; \quad=191 \pm 10.7(5.6 \%) ; \Delta=193 \pm 10.8(5.6 \%) ; \bullet=24 \pm 2.3(9.8 \%)$.] Segments for analysis were approximately $30 \mu \mathrm{m}$ long and weighed $20-40 \mathrm{ng}$. Mean activity ( $\mathrm{mol} / \mathrm{kg}$ dry weight/hr), SD, and cocfficient of variation (in parentheses) are given for each fiber. (Arrow indicates a missing segment.)

If so, the observed biochemical uniformity also provides strong evidence for intrafiber uniformity along the entire length of any fiber.

The uniformity of individual motor units is superimposed upon diverse enzyme levels among different motor units. Data from the 10 motor units in Table 1 plus the 16 random fibers

Table 2. Consistent enzyme activities within an individual motor unit with extreme differences in fiber size

\begin{tabular}{lllll} 
Fiber & $\begin{array}{l}\text { Diameter } \\
(\mu \mathrm{m})\end{array}$ & $\begin{array}{l}\text { Dry weight } \\
(\mathrm{ng})\end{array}$ & $\begin{array}{l}\text { Adenyl- } \\
\text { okinase } \\
(\mathrm{mol} / \mathrm{kg} \text { dry } \\
\mathrm{wt} / \mathrm{hr})\end{array}$ & $\begin{array}{l}\text { Lactate } \\
\text { dehydro- } \\
\text { genase } \\
(\mathrm{mol} / \mathrm{kg} \text { dry } \\
\mathrm{wt} / \mathrm{hr})\end{array}$ \\
\hline 1 & $62 \pm 6$ & $21 \pm 1$ & $212 \pm 30$ & $248 \pm 17$ \\
2 & $56 \pm 2$ & $20 \pm 1$ & $187 \pm 15$ & $252 \pm 17$ \\
3 & $44 \pm 3$ & $14 \pm 1$ & $203 \pm 19$ & $246 \pm 21$ \\
4 & $43 \pm 7$ & $13 \pm 1$ & $193 \pm 7$ & $240 \pm 11$
\end{tabular}

All values are means \pm SD for triplicate determinations on each fiber.

The variability among the four fibers, expressed as coefficient of variation and $F$ value, is $5.5 \%$ and 0.95 for adenylokinase and $2.0 \%$ and 0.26 for lactate dehydrogenase. presented in Figure 4, taken together to represent the fiber population of the whole muscle, have the following ranges of enzyme activities: adenylokinase, 25-246; lactatc dchydrogenase, 24319; malate dehydrogenase, 2.8-17.3, and $\beta$-hydroxyacyl-CoA dehydrogenase, $0.87-10.0 \mathrm{~mol} / \mathrm{kg}$ dry weight $/ \mathrm{hr}$. The FF group of motor units in Table 1 is sufficiently large for evaluation of enzyme variation within a major fiber type. Enzyme ranges are as follows: adenylokinase, 201-246; lactate dehydrogenase, 160312; malate dehydrogenase, 3.1-6.4; and $\beta$-hydroxyacyl-CoA dehydrogenase, $1.3-2.6 \mathrm{~mol} / \mathrm{kg}$ dry weight $/ \mathrm{hr}$. Thus, enzyme variations of up to 2.1-fold occur among fibers that are the same type and, thus, have the same histochemical staining, but belong to different motor units. Coefficients of variation are calculated for each enzyme in Table 1 and are considerably higher in the fiber-type group as a whole than in any of the individual motor units.

\section{Discussion}

Four enzymes representing energy pathways that help characterize muscle fiber specialization (e.g., Burke, 1981; Lowry et al., 1978) were found to be quantitatively identical within individual motor units of cat tibialis posterior muscle. Moreover, this enzymatic uniformity is evidently maintained in individual fibers throughout their length, despite changes in fiber diameter. 
The judgment of homogeneity was based on variance analysis of the inter- and intrafiber enzyme differences. The latter value combines the analytical error of the method and the biological variation along the single fiber. The low $F$ ratios obtained indicate considerable uniformity within the limits of the analytical methods. An $F$ ratio of 1.0 indicates that there was no more difference among fibers than within a single fiber. These findings support earlier quantitative results of motor unit uniformity of rat extensor digitorum longus muscle (Nemeth et al., 1981). A representative unit from the previously reported data was subjected to analysis of variance to determine the variation among and between fibers, and yielded an $F$ ratio of 0.38 for malate dehydrogenase activity, which is in general agreement with the $0.17-3.31$ range for malate dehydrogenase in this report. A similar analysis was also applied to motor units that were newly formed following nerve crush, resulting in similarly low variability between fibers for malate dehydrogenase ( $F$ ratios $0.6-$ 3.4 ), but slightly greater variability in other enzymes (Nemeth and Turk, 1984).

Motor unit uniformity is most important in light of the considerable biochemical heterogeneity of fibers lying close together in a given muscle. Laboratories engaged in single-cell biochemical analysis have shown extreme variations across the different fiber types (Hintz et al., 1980; Lowry et al., 1978; Nemeth and Turk, 1984; Nemeth et al., 1981; Spamer and Pette, 1977, 1979). In fact, variations of over threefold have been reported for oxidative enzymes among type I fibers (generally analogous to type $S$ fibers) of rabbit soleus (Spamer and Pette, 1977, 1979) and over threefold for laclate dehydrogenase among type IIA fibers (similar but not analogous to FR fibers) and IIB fibers (similar to FF fibers) of rat hindlimb muscles (Hintz et al., 1980). In a recent report (Nemeth and Turk, 1984), variance analysis made on about 20 single fibers from each of the major fiber types in rat muscle gave average $F$ ratios of 33 for malate dehydrogenase, 37 for lactate dehydrogenase, and 31 for adenylokinase. The large $F$ ratios indicated significant variations within the fibertype groups and for all of the enzymes measured.

The expanding magnitude of enzyme variations from cell to whole muscle can be exemplified in the present experiments with lactate dehydrogenase. The average coefficient of variation for duplicate determinations from all 80 single fibers was $5.3 \%$ (data not shown); the average variation for the eight fibers of six individual FF motor units was 3.6\% (Table 1); the variation for the six FF typc motor units was $21.2 \%$ (Table 1), and for the 10 motor units in the whole muscle, $48.6 \%$ (Table 1 ). Thus, there is no difference between the enzyme measurements in individual fibers and motor unit fibers, considerable difference between different motor units of the same type, and even more difference across the three fiber types.

A recent report has introduced evidence of variability within motor units with respect to fiber size, alkaline myosin ATPase, succinate dehydrogenase, and $\alpha$-glycerophosphate dehydrogenase staining properties (Roy et al., 1984). Those findings (1) bring forward an important limitation of the glycogen depletion method and (2) draw a distinction in the means of selecting fibers for analysis. Our histochemical evaluation also indicated discrepancies in the depletion patterns: In some cases, entire fascicles composed of fibers of all types were depleted, possibly as a result of local ischemia (Segal and Faulkner, 1985). There were also occasional instances of depleted fibers from myosin ATPase types different from the motor unit. This observation was also made by Kugelberg (1973). Moreover, aberrant depleted fibers were found in control muscles in which nerve stimulation was not applied. Therefore, our strategy was to ensure a priori that the depleted fiber was of the appropriate type. In this way, many fibers could be eliminated from further biochemical analysis based on the histochemistry. It is possible that this selection step accounts for the uniformity found in the present experiments, and their disagreement with the report of Roy et al. (1984). In addition, it should be emphasized that the analytical techniques used to measure enzymes differed substantially: The present method is microanalytical biochemistry, whereas Roy et al. (1984) used computerized photodensitometry of enzyme staining reactions, modified from the methods of Pette et al. (1979). Furthermore, there were undoubtedly operational differences in techniques of glycogen depletion, periodic acid-Schiff reaction, and myosin ATPase staining.

It is evident from Table 1 that general functional relationships exist between physiology and metabolic capacity. As a group, fibers of fast-contracting motor units are much higher in adenylokinase activity than are the slow-contracting ones, presumably because of the need of fast fibers to maximize the availability of ATP. Fast-contracting motor units have a higher glycolytic capacity, as is evident from the higher lactate dehydrogenase activities in that group. Fatigable units, type FF, have lower oxidative enzyme activities for both carbohydrate and fatty acid metabolism than either the $S$ or FR units, which resist fatigue. Numerous other enzymes and physiological parameters are currently under investigation in studies designed to gain further information about the underlying biochemical nature of different motor units and so account for their unique physiological capabilities.

\section{References}

Brooke, M. S., and K. K. Kaiser (1970) Three myosin adenosine triphosphatase systems: The nature of their $\mathrm{pH}$ lability and sulfhydryl dependence. J. Histochem. Cytochem. 18: 670-672.

Buller, A. J., J. C. Fccles, and R. M. Eccles (1960) Interactions between motoneurones and muscles in respect of the characteristic speeds of their responses. J. Physiol. (Lond.) 150: 417-439.

Burke, R. E. (1981) Motor units: Anatomy, physiology and functional organization. In Handbook of Physiology, Vol. II, The Nervous System: Motor Control, Pt. 1, B. Brooks, ed., pp. 345-422, American Physiological Society, Bethesda, MD.

Burke, R. E., D. N. Levine, F. E. Zajac, P. Tsairis, and W. K. Engle (1971) Mammalian motor units: Physiological-histochemical correlation in three types in cat gastrocnemius. Science 174: 709-712.

Edström, L., and E. Kugelberg (1968) Histochemical composition, distribution of fibers and fatigability of single motor units. J. Neurol. Neurosurg. Psychiatry 31: 424-433.

Essén, B., E. Jansson, J. Henriksson, A. W. Taylor, and B. Saltin (1975) Metabolic characteristics of fiber types in human skeletal muscle. Acta Physiol. Scand. 95: 153-165.

Hintz, C. S., C. V. Lowry, K. K. Kaiser, D. McKee, and O. H. Lowry (1980) Enzyme levels in individual rat muscle fibers. Am. J. Physiol. 239 (Cell Physiol. 8): C58-C65.

Hintz, C. S., M. M.-Y. Chi, and O. H. Lowry (1984) Heterogeneity in regard to enzymes and metabolites within individual muscle fibers. Am. J. Physiol. 246 (Cell Physiol. 15): C288-C292.

Krnjevic, K., and R. Miledi (1958) Motor units in the rat diaphragm. J. Physiol. (Lond.) 140: 427-439.

Kugelberg, E. (1973) Histochemical composition, contraction speed and fatigability of rat soleus motor units. J. Neurol. Sci. 20: 177-198.

Lowry, O. H., and J. V. Passonneau (1972) A Flexible System of Enzymatic Analysis, Academic, New York.

Lowry, C. V., J. S. Kimmey, S. Felder, M. M.-Y. Chi, K. K. Kaiser, P. N. Passonneau, K. A. Kirk, and O. Lowry (1978) Enzyme patterns on single human muscle fibers. J. Biol. Chem. 253: 8269-8277.

McDonagh, J. C., M. D. Binder, R. M. Reinking, and D. G. Stuart (1980) Tetrapartite classification of the motor units of cat tibialis posterior. J. Neurophysiol. 43: 621-629.

Nemeth, P. M., and W. R. Turk (1984) Biochemistry of single fibers in newly assembled motor units following nerve crush. J. Physiol. (Lond.) 355: 547-555.

Nemeth, P. M., D. Pette, and G. Vrbová (1981) Comparison of enzyme activities among single muscle fibers within defined motor units. $J$. Physiol. (Lond.) 311: 489-495.

Nemeth, P. M., J. L. Park, D. G. Stuart, R. M. Reinking, L. Rankin, S. Vanden-Noven, and T. M. Hamm (1984) Physiological and bio- 
chemical properties of individual motor units of cat muscle. Soc. Neurosci. Abstr. 10: 781.

Nemeth, P. M., T. M. Hamm, D. A. Gordon, R. M. Reinking, and D. G. Stuart (in press) Application of cross-sectional single-fiber microchemistry to the study of motor unit fatigability. In Motor Control, G. N. Gantchev, B. Dimitrov, and P. Gatev, eds., Plenum, New York.

Pette, D., H. Wasmund, and M. Wimmer (1979) Principle and method of kinetic microphotometric enzyme activity determination in situ. Histochemistry 64: 1-10.

Roy, R. R., S. C. Martin, E. Bodine, E. Eldred, and V. R. Edgerton (1984) Fiber size and metabolic variability within cat tibialis anterior motor units. Soc. Neurosci. Abstr. 10: 73.

Segal, S. S., and J. J. Faulkner (1985) Temperature-dependent physiological stability of rat skeletal muscle in vitro. Am. J. Physiol. 248 (Cell Physiol. 17): C265-C270.

Sherrington, C. S. (1925) Remarks on some aspects of reflex inhibition. Proc. K. Soc. Lond. [Biol.] 97: 519-545.
Spamer, C., and D. Pette (1977) Activity patterns of phosphofructokinase, glyceraldehyde-phosphate dehydyrogenase, lactate dehydrogenase and malate dehydrogenase in microdissected fast and slow fibres from rabbit psoas and soleus muscle. Histochemistry 52: 201216.

Spamer, C., and D. Pette (1979) Activities of malate dehydrogenase, 3-hydroxyacyl-CoA dehydrogenase and fructose-1,6-diphosphate with regard to metabolic subpopulations of fast and slow twitch fibres in rabbit muscle. Histochemistry 60: 9-19.

Stuart, D. G., M. D. Binder, and R. M. Enoka (1984) Motor unit organization: Application of the quadrapartite scheme to human muscles. In Peripheral Neuropathy, Vol. 1, P. J. Dyck, P. K. Thomas, E. H. Lambert, and R. P. Bunge, eds., pp. 1067-1090, Saunders, Philadelphia.

Vrbová, G., T. Gordon, and R. Jones (1978) Nerve-Muscle Interaction, pp. 53-118, 166-181, Chapman and Hall, London. 